\title{
OSI Open Knowledge Stakeholder Group Report
}

Cheryl Ball, Sioux Cumming, Stacy Konkiel, Joan Lippincott, David Mellor, Kamran Naim, Jake Orlowitz, Louise Page, Richard Price, Jason Steinhauer, Mary Yess

\section{Summary of Discussions}

The Open Knowledge Stakeholder group was heterogeneous, with representation from nonprofit societies, academia, new OA journals, and service providers in most aspects of the scholarly communications lifecycle spectrum. There were many varied activities reported, as well as an array of opinions and comments; however, some main conclusions emerged.

- The ideal scenario for "open" is "free-free open," i.e. free to publish and free to consume.

- There is no need for one model only; there are many ways to accomplish open.

- This is an exciting, and good, time to experiment.

- The common strategy is to transition into full openness.

- Establishing financial sustainability for a "free-free" environment is the true challenge.

- There is a need to get content to the communities who would benefit most from it.

- Greater uptake in the general community for openness is needed. All stakeholders should communicate without jargon, which is a barrier to understanding.

- In addition to open access, there are concerns related to openness in oth- er parts of the scholarly communication ecosystem that must inform these discussions - transparency, reproducibility, incentives, peer review, etc.- - from the conception of a study and its methodology to the raw data and published results.

\section{Recommendations}

The second session of this group was sparsely attended, because people attended other groups during the mix-ups; and because the structure of this stakeholder meeting was not as obvious as that of the workgroups. The following was the consensus of the group's second session.

- Our time, thoughts, and efforts were/are going into our workgroups, not the stakeholder groups - there's just not enough time and energy to do both at the conference and afterward.

- Stakeholder groups were great meetand-greet/networking sessions, but it was very difficult to organize in such a short timeframe because the theme, at least for this group, was so very broad, that most of the time was spent "getting started." Thus there wasn't time to come to substantive conclusions and/or recommendations.

(C) 2017 OSI2017 Open Knowledge Stakeholder group. This open access article is distributed under the Creative Commons Attribution 4.0 International License. This document reflects the combined input of the authors listed here (in alphabetical order by last name) as well as contributions from other OSI2017 delegates. The findings and recommendations expressed herein do not necessarily reflect the opinions of the individual authors listed here, nor their agencies, trustees, officers, or staff. 
- During the sessions, no leader for the group self-identified, so participants reported on what their respective organizations were doing; this took up most of the first session, with some discussion in between. This was a very free-form affair and the discussion from the first session was not captured. The planning committee might consider this issue (leadership in these groups), and might consider a different process for identifying a leader, even if only to establish some kind of "starter" leader.

- Stakeholder meetings might not be outcome-oriented (no reports) and these timeslots could be used as replacements for the workgroup mixups. This would give delegates the opportunity to share reflections, commonalities, and serve as a clearinghouse for information.

- The Open Knowledge group might need further delineation, as most of the people in this group could be part of many other stakeholder groups. After all, isn't all of OSI about "open knowledge"?

- Given the above comments, and those of other stakeholder groups, the planning committee might want to consider disbanding the stakeholder groups.

\section{Perspectives of the Group's Par- ticipants}

Altmetric http://www.altmetric.com

Altmetric's mission is to track and analyse the online activity around scholarly research outputs. We are one of several companies that provide altmetrics, which are data from the social web that helps researchers, university administrators, publishers, and funders understand where and how research is discussed online.

Much of the conversation in our stakeholder group meeting at OSI 2017 concerned incentives and how metrics can drive researchers' behavior, for better or for worse. We believe that by providing more diverse research impact metrics to academia, we can incentivize researchers to take up behaviors that benefit all: increased public engagement, open access publishing, data sharing, and more.

\section{Center for Open Science \\ https://cos.io}

Our mission is to increase openness, integrity, and reproducibility of research. These are core values of scholarship and practicing them is presumed to increase the efficiency of acquiring knowledge.

For COS to achieve our mission, we must drive change in the culture and incentives that drive researchers' behavior, the infrastructure that supports their research, and the business models that dominate scholarly communication.

This culture change requires simultaneous movement by funders, institutions, researchers, and service providers across national and disciplinary boundaries. Despite this, the vision is achievable because openness, integrity, and reproducibility are shared values, the technological capacity is available, and alternative sustainable business models exist.

COS's philosophy and motivation is summarized in its strategic plan and in scholarly articles outlining a vision of scientific utopia for research communication and research practices. 
Because of our generous funders and outstanding partners, we are able to produce entirely free and open-source products and services. Use the header above to explore the team, services, and communities that make COS possible and productive.

\section{Coalition for Networked Information (CNI)}

https://www.cni.org

The Coalition for Networked Information (CNI) is dedicated to supporting the transformative promise of digital information technology for the advancement of scholarly communication and the enrichment of intellectual productivity. Some 230 institutions representing higher education, publishing, information technology, scholarly and professional organizations, foundations, and libraries and library organizations make up CNI's members. Semi-annual membership meetings bring together representatives of CNI's constituencies to discuss ongoing and new projects, and to plan for future initiatives. One of our three main program areas is "Developing \& Managing Networked Information Content," and themes within that area for the current program year are Institutional and Disciplinary Implications of E-Research, Digital Preservation, and Institutional Content Resources and Repositories.

\section{Digital Publishing Institute \\ http://dpi.lib.wvu.edu}

The Digital Publishing Institute (DPI) is an international institute for digitally oriented research that focuses on publishing open-access (OA) scholarship.

The DPI is housed within the West Virginia University (WVU) Libraries and supports faculty, staff, and student pro- duction of publishing projects that require digital and media-rich components. As a new institute within WVU Libraries, the DPI is part of an organization experiencing rapid change.

Over the next few years, we will be working to establish an innovative hub for scholarly communications within the WVU community that will provide a growing number of hosted research projects and OA journal hosting as well as classes, presentations, workshops, and summer institutes highlighting the publishing capacity of scholars using digital tools and technologies.

Three of our current projects include building the Vega academic publishing platform (funded through the Andrew W. Mellon Foundation) to create and host open-access, multimedia-driven research content; teaching KairosCamp (funded through $\mathrm{NEH}$ ) to authors interested in designing scholarly multimedia research such as born-digital humanities projects; and "Many Voices: Building a Consortium of Small Scholarly Societies in the $\mathrm{Hu}-$ manities," a planning grant also funded by the Mellon Foundation to research the provision of shared human and technical infrastructures for small, scholarly societies so that can then focus on flipping their journals to open access.

\section{ECS}

\section{http://electrochem.org}

Electrochemistry and solid state science are the future: they are the leading sciences that will ensure our survival on this planet. ECS believes that by opening and democratizing research, we can more rapidly advance our important sciences and society at large, while directly fulfilling our mission. The key to scientific advancement has always been the open exchange of information. Yet even in today's digital 
environment, many scientists around the world struggle to access quality, reliable research. The bottom line is discoveries need discoverability and that is only guaranteed through full open access.

By creating uninhibited availability of the science, ECS can "free the science" and accelerate scientific discovery and innovation, leading the community as the advocate, guardian, and facilitator of our technical domain.

Free the Science is a bold, long-term vision to further ECS's mission to disseminate and advance our fields by embracing a more open science paradigm to promote innovation. It is a business-model changing initiative that will make our research freely available to all readers, while remaining free for authors to publish. This initiative can set a new publishing standard for ECS (and others), one of the last independent scientific society publishers.

As ECS develops its vision for a shift toward more open science, the Society is becoming more involved in the open community and serving as an advocate for the physical sciences as the inevitable changes take place in scholarly publishing. In 2017, ECS will hold its first satellite OpenCon on open science, host a data sciences hack day, launch a preprint server, and a new "born OA" journal.

\section{History Communication}

http://www.historycommunication.com

The \#histcomm movement aims to ensure that historical scholarship gets communicated effectively to non-experts across the wide array of media available today and in the future.

At the heart of the movement is a desire to see that scholarship by historians pro- duced in academic journals and scholarly monographs is made more visible, accessible and understandable to audiences that do not have the same subject matter expertise.

In some ways, the need for \#histcomm is a work-around for a lack of open access. Virtually no one outside of academic historians reads the thousands of research articles and monographs produced by historians annually. It is inaccessible to most people, and also largely unintelligible. \#Histcomm asks the question of what would happen if historians (and others) repackaged that same scholarship as videos, podcasts, memes, gifs, emojis, snapchats and more, and disseminated it via new technologies. Would more scholarship reach wider audiences? Would that improve public understandings of history, which often lag many years behind the academy?

\#Histcomm also asks the question of what training and instruction are needed for historians to do this work. In addition to the research and analysis skills taught to historians in undergraduate and graduate programs, should we also be teaching communications strategies and media literacy--and specifically applying these skills to the dissemination of historical arguments?

Being part of the OSI conversation is vital for \#histcomm. If history journals transition to open, this will shift how \#histcomm functions and what changes to the history profession it should aim to inspire. Perhaps the model would shift from re-packaging scholarship that most people cannot access, to directing people to scholarship which is freely open and available. Whatever the future, \#histcomm 
wishes to remain part of the conversation and a participant in shaping the culture of open and scholarly communication more generally.

\section{INASP}

http://www.inasp.info/

INASP is an international development charity working with a global network of partners in Africa, Latin America and Asia. We believe that research and knowledge have a crucial role to play in addressing global challenges and contributing to the achievement of the Sustainable Development Goals. To realize this potential, we work in partnership to strengthen the capacity of individuals and institutions to produce, share and use research and knowledge, in support of national development.

INASP works with publishers to enable affordable and sustainable access to online resources to developing countries in Africa, Asia and Latin America. We work with national consortia or equivalent bodies so that they can meet the information needs of their researchers. The Journals Online project aims to improve the accessibility and visibility of developing-country research by providing a cost-effective and secure platform for online journals. AuthorAID is a free pioneering global network that provides support, mentoring, resources and training for researchers in low and middle income countries.

\section{The Open Access Publishing Cooperative Project: A Stanford University \& Public Knowledge Project Initiative http://oa-cooperative.org} The Open Access Cooperative Project, supported by the John D. and Catherine
T. Macarthur Foundation, is investigating the potential for collective and cooperative models in which libraries and publishers work together to develop economically responsible and sustainable paths to open access to rigorously reviewed and professionally published research. We are exploring this premise through two major initiatives. The first, LIBRARIA, is a collective of anthropology, archaeology and social studies of science journals and learned societies that have teamed with the PKP and the Scholarly Publishing and Academic Resources Coalition (SPARC) to develop a cooperative alternative to the subscription economy to advance $\mathrm{OA}$ within these disciplines, which are not well supported by existing APC-based models for OA. Next, we are examining the feasibility of a "subscription-equivalent" transition to open access, leveraging the existing resources and mechanisms of the subscription economy, but repurposing library subscription spends on journals from limited-access subscriptions, to supporting the publishing of content open access, through an approach that is not based on the payment of APCs. This model is revenue neutral for publishers, and expenditure neutral for libraries, i.e. for the cost of a subscription to a journal(s), libraries are able to serve not only the needs of their users and patrons, but to deliver scholarship globally by financing OA publishing of content.

We are in the process of testing the principles and paths for implementation of this model through surveying the library community, as well as extensive consultations with a broad range of scholarly publishers in order to understand the opportunities and pitfalls of this approach. We are testing implementation of this model in cooperation with the non-profit publisher Annual Reviews, who, with the 
support of the Robert Wood Johnson Foundation have transitioned 2017 volume of the Annual Review of Public Health to OA, with all previous volumes now available freely online. Annual Reviews is in the process of testing the col- lective model, starting with subscribing libraries, to gain the support of libraries to redirect their subscription spend towards a collective fund to publish subsequent volumes of the journal OA.

\section{Open Knowledge Stakeholder Group:}

Cheryl Ball, Director, Digital Publishing Institute, West Virginia University

Sioux Cumming, Programme Manager Journals Online, INASP

Stacy Konkiel, Director of Research and Education, Altmetric.com

Joan Lippincott, Associate Executive Director, Coalition for Networked Information

David Mellor, Project Manager, Journal and Funder Initiatives, Center for Open Science

Kamran Naim, Lead Researcher, Open Access Cooperative Study, Stanford University; Strategic Development Manager, Annual Reviews

Jake Orlowitz, Head of The Wikipedia Library, Wikimedia Foundation

Louise Page, Publisher, PLOS

Richard Price, Founder and CEO, academia.edu

Jason Steinhauer, Director, Lepage Center for History in the Public Interest, Villanova University

Mary Yess, Deputy Executive Director \& Chief Content Officer, The Electrochemical Society 